To appear in: Proc. CVPR'93

\title{
Toward Global Surface Reconstruction By Purposive Viewpoint Adjustment
}

\author{
Kiriakos N. Kutulakos \\ Charles R. Dyer \\ kyros@cs.wisc.edu \\ dyer@cs.wisc.edu \\ Computer Sciences Department \\ University of Wisconsin \\ Madison, Wisconsin 53706 USA
}

\begin{abstract}
We consider the following problem: How should an observer change viewpoint in order to generate a dense image sequence of an arbitrary smooth surface so that it can be incrementally reconstructed using the occluding contour and the epipolar parameterization? We present a collection of qualitative behaviors that, when integrated appropriately, purposefully control viewpoint based on the appearance of the surface in order to provably solve this problem.
\end{abstract}

\section{Introduction}

What qualitative, real-time behaviors [1] are important for performing global, geometric tasks such as searching for specific surface markings (e.g., the bar code on a grocery store item), building a global model of an object, or recognizing an object? Clearly, a general approach to these tasks requires the ability to purposefully control the point of observation based on the appearance of the object.

In this paper we combine the shape-from-motion paradigm with the purposive vision paradigm [2] to develop a collection of viewpoint control behaviors for deriving a global, threedimensional description of an arbitrary object. The behaviors we describe address the following problem: How should the motion of the observer be controlled in order to generate a dense sequence of images that allows global reconstruction of the surface using the occluding contour and the epipolar parameterization [3]? Our behaviors are developed in the context of three increasingly more general reconstruction tasks: The local reconstruction task, where the observer must control viewpoint to reconstruct a patch around a selected surface point; the incremental reconstruction task, where the observer must control viewpoint to iteratively "grow" a reconstructed region on the surface; and the global reconstruction task, where an entire reconstructible region on the surface must be reconstructed. All these tasks require the use of multiple viewpoint control behaviors that must be appropriately integrated. Previous work on shape-from-motion has not considered these tasks, assuming the observer's motion is independent of the scene. Below we describe a collection

The support of the National Science Foundation under Grant No. IRI-9022608 is greatfully acknowledged. of behaviors that provably perform the first two tasks for a (generic or algebraic) smooth surface of arbitrary shape; the surface is unknown, can be non-convex, and can self-occlude. In [4] we show how these behaviors can be used to perform the global reconstruction task.

\section{Local Reconstruction by Purposive Motion}

In our approach, both global and incremental reconstruction is performed by integrating a collection of qualitative viewpoint control behaviors, each of which performs an instance of the following local reconstruction task: Suppose the observer is at position $c$, and let $p$ be a visible rim point on the surface identified by its projection, $p_{\text {oce }}$, on the occluding contour. The observer's task is to continuously control viewpoint, starting at $c$, in order to recover the shape of the surface for all points in some neighborhood of $p$ using the epipolar parameterization. Given a collection of basic behaviors that provably perform this task, incremental surface reconstruction is achieved by appropriately combining them (see Section 3 ).

The visible rim is the one-dimensional set of visible points on the surface whose tangent plane contains the line connecting these points to the observer's viewpoint. The occluding contour is the projection of the visible rim on the image. The epipolar parameterization captures the idea that under continuous motion of the observer (and when the topology of the occluding contour does not change), the set of points comprising the visible rim consists of smooth curves that "slide" over the surface. This allows the reconstructible points on the surface (i.e., the non-concave surface points that can lie on the visible rim) to be considered as a collection of patches, each of which is a family of visible rim curves [3].

Our approach is based on the following three observations. First, if $p$ is not the endpoint of a visible rim curve and the visible rim's topology does not change in the neighborhood of $p$ if the observer's viewpoint is infinitesimally perturbed, the observer's motion can be controlled so that there exists a neighborhood $\Pi$ of $p$ such that the surface in $\Pi$ can be described by the epipolar parameterization. Second, if $p$ is the endpoint of a visible rim curve, the epipolar parameterization cannot describe the surface in the neighborhood of $p$. However, there are other viewpoints on $p$ 's tangent plane at 
which $p$ is not the endpoint of a visible rim curve. Third, $p$ and the observer's viewpoint may be such that the occluding contour's topology changes in the neighborhood of $p$ under an infinitesimal viewpoint perturbation. For all points $p$ except those lying on a collection of special sets (which are curves for generic surfaces), the observer can move to other viewpoints on $p$ 's tangent plane at which the contour's topology does not change in the neighborhood of $p$ if these viewpoints are infinitesimally perturbed. The special sets for which this cannot be achieved bound the reconstructible surface regions [4].

Based on these observations, for any given viewpoint $c$ we distinguish four types of visible rim points, corresponding to the four instances of the local reconstruction task: Ordinary points, which are not endpoints of a visible rim curve; cusp points, which are visible rim endpoints projecting to a cusp on the occluding contour at $c ; T$-junction points, which are visible rim endpoints projecting to a T-junction on the occluding contour at $c$; and degenerate points, where the occluding contour's topology changes in the vicinity of their projection if $c$ is infinitesimally perturbed. The basic behavior we use for performing the local reconstruction task controls the motion of the observer in order to deal with the case where $p$ is an ordinary visible rim point; the other three cases are treated by (1) controlling the position of the observer in order to reach a viewpoint where $p$ is an ordinary visible rim point, and (2) applying the basic step in order to recover the shape of the surface in a neighborhood of that point. We present only two of the four behaviors. The interested reader is referred to [4].

\subsection{Reconstruction Around Ordinary Points}

Suppose the observer changes viewpoint by tracing a smooth curve $c(t)$, and let $\mathbf{v}(t)=c^{\prime}(t)$ be the instantaneous direction of motion. Given a segment $\beta(t)$ of the visible rim at viewpoint $c(t)$, the epipolar parameterization allows us to define the segment $\beta(t+\delta t)$ of the visible rim at $c(t+\delta t)$ that corresponds to $\beta(t)$. The following theorem immediately suggests a simple, qualitative behavior for controlling the motion of the observer to solve the local shape recovery problem for a selected ordinary visible rim point $p$ [4]:

Theorem 1 If $\beta(t)$ is a visible rim segment whose points $q$ satisfy $\mathbf{n}(q) \cdot \mathbf{v}(t)>0$, then all points of $\beta(t+\delta t)$ are occluded from position $c(t)$.

\section{Ordinary Patch Reconstruction Behavior}

Step 1: Select a point $p$ on the visible rim that is not the endpoint of a visible rim curve. This is done indirectly by selecting $p$ 's projection, $p_{o c c}$, on the occluding contour. Point $p_{o c c}$ must not be the endpoint of an occluding contour curve.

Step 2: Compute the surface normal at $p$. It is given by $\mathbf{T} \wedge p_{o c c}$, where $\mathbf{T}$ is the tangent to the occluding contour at $p_{\text {occ }}$.

Step 3: (Reconstructing the occluded points near $p$.) Select a motion direction $\mathbf{v}_{1}$ satisfying $\mathbf{n}(p) \cdot \mathbf{v}_{1}>0$. Change viewpoints along $\mathbf{v}_{1}$ while continuously monitoring the deformation of the occluding contour curve that initially contains $p_{\text {occ }}$.

Step 4: (Reconstructing the visible points near $p$.) Move back to the initial viewpoint and reapply Step 3 by selecting another direction of motion $\mathbf{v}_{2}$ satisfying $\mathbf{n}(p) \cdot \mathbf{v}_{2}<0$.

\subsection{Reconstruction Around Cusp Points}

Let $p_{o c c}$ be the projection of a point $p$, and let $C(s)$ be the occluding contour curve containing $p_{o c e}$, with $p_{o c c}=C\left(s_{0}\right)$. The following theorem characterizes the special directions for changing the observer's viewpoint that force $p$ to become ordinary, and leads to a qualitative behavior for controlling viewpoint to reconstruct the surface around cusp points:

Theorem 2 Let $p_{\text {oce }}$ be the projection of a cusp point $p$ on the visible rim, and let $\mathbf{T}=\lim _{s \rightarrow s_{0}} C^{\prime}(s)$. If the observer performs an infinitesimal viewpoint change along a direction $\mathbf{v}$ in $p$ 's tangent plane such that $\mathbf{v} \cdot \mathbf{T}>0, p$ will be an ordinary visible rim point at the new viewpoint.

\section{Cusp Patch Reconstruction Behavior}

Step 1: Compute the tangent $T$ to the occluding contour at the selected occluding contour cusp endpoint $p_{\text {occ }}$.

Step 2: Compute the normal $\mathbf{n}(p)$ of the surface at the corresponding visible rim point $p$.

Step 3: Determine whether a clockwise or counterclockwise direction of motion $\mathbf{v}$ around $p$ on $p$ 's tangent plane satisfies the inequality $\mathbf{v} \cdot \mathbf{T}>0$.

Step 4: Perform a small viewpoint change in the direction selected at Step 3 while fixating at $p$.

Step 5: Use the Ordinary Patch Reconstruction Behavior to control viewpoint for reconstructing a surface patch around $p$.

\section{The Incremental Reconstruction Behavior}

The surface can now be incrementally reconstructed by integrating the four behaviors used for the local reconstruction task in the following way:

Step 1: If there exists a portion of the surface that has not been reconstructed, select a point $p$ on its boundary and let $c$ be the viewpoint at which $p$ projected to the occluding contour.

Step 2: Move to $c$, use the viewpoint control behavior appropriate for performing the local reconstruction task around $p$, and continue with Step 1.

\section{References}

[1] D. H. Ballard and C. M. Brown, "Principles of animate vision," CVGIP: Image Understanding, v. 56, n. 1, pp. 3-21, 1992.

[2] Y. Aloimonos, "Purposive and qualitative active vision," in Proc. ICPR pp. 346-360, 1990.

[3] R. Cipolla and A. Blake, "Surface shape from the deformation of apparent contours," IJCV , v. 9, n. 2, pp. 83-112, 1992.

[4] K. N. Kutulakos and C. R. Dyer, "Global surface reconstruction by purposive viewpoint adjustment,” TR1141, CS Dept., University of Wisconsin - Madison, April 1993. Available via ftp from ftp.cs.wisc.edu. 